\title{
Accessory Cardiac Conduction Pathway with an Unusual Presentation
}

\author{
Ikram Ahmed Rana and Wajeeha Afzal \\ Department of Cardiology, Tahir Heart Institute, Chenab Nagar, District Chiniot, Pakistan
}

\begin{abstract}
Pre-excitation syndrome (PES) is a congenital abnormality in which there is conduction through accessory pathway in addition to atrioventricular (AV) node between the atria and the ventricles. Conduction through accessory pathway is without any delay and; hence, results in early excitation of ventricles. This dual connection provides substrate for atrioventricular reciprocating tachycardia (AVRT), a type of supra-ventricular tachycardia. Wolf-Parkinson-White (WPW) syndrome is the commonest form of PES. PES with third degree AV block or complete heart block $(\mathrm{CHB})$ is a rare association. We report here a case of 37-year male with surface ECG findings consistent with PES who presented with syncope. The combination of pre-excitation on ECG with syncope usually draws attention towards tachy-arrhythmias. However, ECG monitoring revealed CHB with intermittent conduction through accessory pathway. It highlights the critical evaluation of PES patients with syncope because therapeutic strategy is entirely different. This case was managed with dual chamber pacemaker implantation instead of accessory pathway ablation.
\end{abstract}

Key Words: Atrioventricular block, Pre-excitation syndrome, Syncope.

How to cite this article: Rana IA, Afzal W. Accessory cardiac conduction pathway with an unusual presentation. J Coll Physicians Surg Pak 2019; 29 (Supplement 2):S77-S79.

\section{INTRODUCTION}

Pre-excitation syndrome (PES) is a congenital abnormality of the conducting tissue of heart in which an accessory pathway (AP) is present that can provide electrical connection between atria and ventricles in addition to atrioventricular (AV) node. Changes on surface ECG depend upon the location, origin and insertion of AP. In case of AV connection with antegrade conduction, changes like short PR interval, a delta wave, and a widened QRS complex are seen on surface ECG. These ECG changes together with supra-ventricular tachycardia is referred to as Wolf-Parkinson-White (WPW) syndrome. 1

WPW syndrome is considered to be the classic form of PES. The prevalence of WPW syndrome is 0.7 to 1.7 per 1,000 persons with male predominance. It usually manifests in second and third decade. The risk of sudden cardiac death is less than $1 \% .^{2}$ The commonest arrhythmia in WPW syndrome is atrio-ventricular reciprocating tachycardia (AVRT) with $95 \%$ orthodromic tachycardia. It has been estimated that one-third of patients with WPW syndrome have atrial fibrillation. It can lead to life-threatening ventricular fibrillation. ${ }^{3}$ Management of asymptomatic patients with PES has always remained controversial. Catheter ablation of AP

Correspondence to: Dr. Ikram Ahmed Rana, Department of Cardiology, Tahir Heart Institute, Chenab Nagar,

District Chiniot, Pakistan.

E-mail: ikramahmedrana@gmail.com

Received: November 02, 2018; Revised: April 01, 2019;

Accepted: April 20, 2019 has become an established mode of therapy for symptomatic patients and asymptomatic patients employed in high-risk professions. 4

Third degree AV block or complete heart block (CHB) refers to complete blockage of conduction through the nodal-HIS system leading to electro-mechanical dissociation of atria and ventricles. It can be congenital or secondary to infections, coronary artery disease, cardiomyopathies, autoimmune diseases, degenerative diseases and endocrinological diseases. The prevalence of $\mathrm{CHB}$ is $0.04 \% .^{5}$ Therefore, the association between $\mathrm{CHB}$ and WPW syndrome is extremely infrequent. While reviewing literature, only few cases were found with similar clinical and electrophysiological characteristics. ${ }^{6}$

Usually, syncope in patients with PES is thought to be due to supra-ventricular tachycardia involving AP. But in this case, the mechanism of syncope was $\mathrm{CHB}$ with entirely different management strategy.

\section{CASE REPORT}

A 37-year male was referred from basic health facility in periphery to Tahir Heart Institute, Chenab Nagar, Pakistan on 9th July, 2018 with one episode of syncope. He did not have any significant past medical or surgical history. He was not taking any medicine. He had no history of syncope or palpitation in the past. His general physical and systemic examinations were unremarkable except for bradycardia at 53 beats/minute. ECG at presentation showed pre-excited QRS (Delta waves), short PR (approximately 110ms) and left axis deviation (Figure 1). Further, chest leads showed sudden transition of $R>S$ wave from $V 1$ to $V 2$ suggesting location of $A P$ as 
posterior-septal. With such ECG changes together with history of syncope, the first diagnosis that strikes the mind is tachy-arrhythmia in the form of AVRT or rapidly conducting paroxysmal atrial fibrillation. Patient was kept under observation. Cardiac monitoring revealed episodes of $\mathrm{CHB}$ with intermittent conduction through AP (Figure 2). Heart rate dropped to 30 beats/minute. Temporary pacemaker was inserted immediately. All available investigations for acquired causes of $\mathrm{CHB}$ showed negative results. These included serum angiotensin converting enzyme (ACE) level, serum thyroid function tests, cardiac biomarkers, including troponin I and CKMB and serum potassium level. Echocardiography did not show any structural heart defect. Electrophysiological study was not available. Considering CHB, possibility of AVRT was least likely. On the next day, dual chamber pacemaker was implanted successfully. He was kept under observation for two more days. No complication was noted. No more episodes of syncope occurred and the patient was discharged on $3^{\text {rd }}$ day.

\section{DISCUSSION}

The association between CHB and the WPW syndrome is very infrequent. We have come across few cases in the literature that describe this association. ${ }^{7}$ This patient never experienced a single episode of palpitation in the past. It is known that the conduction properties of the AP worsen with age, the development of tachycardia decreases over time and pre-excitation can even disappear.8 Although conduction through AP is an abnormal occurrence, in this case, it was, in fact, beneficial. During hospital stay, cardiac monitoring revealed very unstable ventricular escape rhythm. It was conduction through AP that saved his life. There is a possibility of isolated congenital CHB which remained masked by conduction through $\mathrm{AP}$ and with time due to worsening of conducting properties of $\mathrm{AP}$, the $\mathrm{CHB}$ manifested. This could be the reason of late presentation of patient. Negative work up for acquired causes of $\mathrm{CHB}$ and age of patient also raise the suspicion of congenital CHB. Irrespective of the cause of $\mathrm{CHB}$, the objective of this case report is to highlight that syncope with PES is not always due to tachyarrhythmias. Secondly, the short-term therapeutic plan for tachy-arrhythmia are rate limiting anti-arrhythmic drugs which could have further worsened the conduction in this case. And long-term plan like AP ablation would certainly knock out conduction between atria and ventricles.

With dysfunctional AV node, the possibility of AVRT was least likely. Though, it cannot be ruled out because very rarely multiple AP's may co-exist. ${ }^{9}$ They can provide substrate for AVRT. Although electrophysiology study was not done in this case, but it was not expected to alter the management plan as CHB was clearly evident

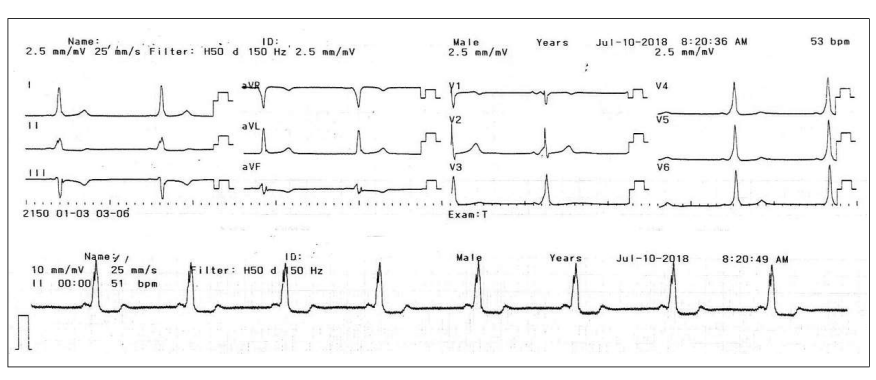

Figure 1: ECG changes of pre-excitation syndrome.

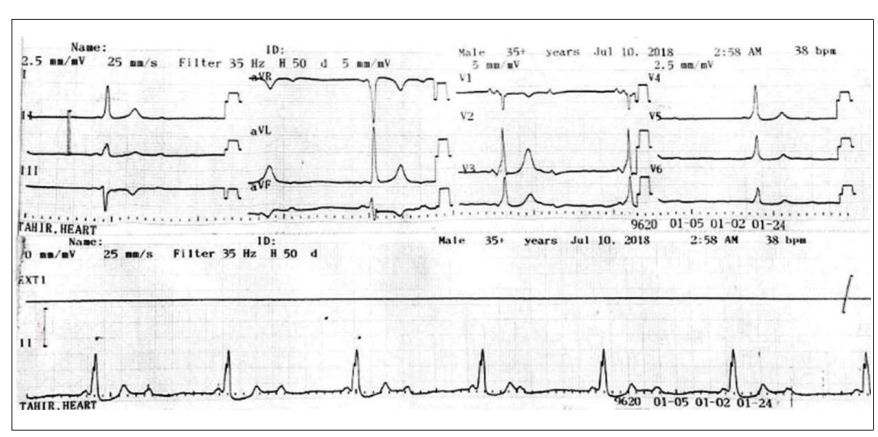

Figure 2: AV nodal conduction block with intermittent conduction through AP.

on cardiac monitoring. This case emphasises the need of careful evaluation of AV node before ablation of AP in WPW syndrome patients. A case has been reported in which nodal-HIS conduction block became evident after ablation of AP.10 It is particularly difficult to unmask the nodal-HIS conduction block in AP's with shorter antegrade effective refractory period as compared to $\mathrm{AV}$ node.

We recommend that cause of syncope must be sought in patients with PES in same way as in other patients. One should not rush for rate limiting antiarrhythmic medications as these can be detrimental to patient. And lastly, AV nodal conduction should be carefully evaluated before AP ablation.

\section{PATIENT'S CONSENT:}

Informed consent was obtained from the patient to publish this case report.

\section{CONFLICT OF INTEREST:}

Authors declared no conflict of interest.

\section{AUTHORS' CONTRIBUTION:}

WA: Involved in writing case report section.

IAR: Reviewed the literature, wrote introduction, discussion sections, and performed proofreading.

\section{REFERENCES}

1. Cay S, Topaloglu S, Aras D. Percutenous catheter ablation of the accessory pathway in a patient with Wolff-Parkinson-White syndrome associated with familial atrial fibrillation. Indian Pacing Electrophysiol J 2008; 8:141-5. 
2. Lu CW, Wu MH, Chen HC, Kao FY, Huang SK. Epidemiological profile of Wolff-Parkinson-White syndrome in a general population younger than 50 years of age in an era of radiofrequency catheter ablation. Int J Cardiol 2014; 174:530-4.

3. Centurión OA, Shimizu A, Isomoto S, Konoe A. Mechanisms for the genesis of paroxysmal atrial fibrillation in the WolffParkinson-White syndrome: Intrinsic atrial muscle vulnerability vs. electrophysiological properties of the accessory pathway. Europace 2008; 10:294-02.

4. Sethi KK, Dhall A, Chadha DS, Garg S, Malani SK, Mathew OP. WPW and pre-excitation syndromes. J Assoc Physicians India 2007; 55:10-15.

5. Kojic EM, Hardarson T, Sigfusson N, Sigvaldason H. The prevalence and prognosis of third-degree atrioventricular conduction block: The Reykjavik study. J Intern Med 1999; 246:81-6.

6. Singh B, Kaul U, Talwar KK, Wasir HS. Coexistence of preexcitation, Mobitz type II Kent bundle block and complete infra-
Hisian block in a patient with syncope. Indian Heart J 1995; 47:146-8.

7. Palanca V, Quesada A, Roda J, Villalba S, Mihi N, Velasco J. Intermittent atrioventricular block in an accessory pathway associated with complete infrahissian block. Rev Esp Cardiol 2004; 57:363-6.

8. Michelucci A, Padeletti L, Mezzani A, Giovannini T, Miceli M, Cupelli $\mathrm{V}$, et al. Relationship between age and anterograde refractoriness of the accessory pathway in Wolff-ParkinsonWhite patients. Cardiology 1989; 76:270-3.

9. Orczykowski M, Derejko P, Urbanek P, Bodalski R, ZakrzewskaKoperska J, Bilinska M, et al. Characteristic features of patients with multiple accessory pathways. Acta Cardiol 2017; 72: 404-9.

10. García Pinilla JM, Barrera A, de Teresa E, Alzueta J. Syncope in a patient with Wolff-Parkinson-White syndrome. Med Clin (Barc) 2003; 120:119. 